\title{
Corela
}

Cognition, représentation, langage

HS-21 | 2017

Linguistique de corpus : vues sur la constitution, l'analyse et l'outillage

\section{Analyse contrastive des patrons verbaux dans l'écrit scientifique entre scripteurs étudiants et experts}

\author{
Sylvain Hatier et Rui Yan
}

\section{OpenEdition}

\section{Journals}

Édition électronique

URL : http://journals.openedition.org/corela/4879

DOI : $10.4000 /$ corela.4879

ISSN : 1638-573X

Éditeur

Cercle linguistique du Centre et de l'Ouest - CerLICO

Référence électronique

Sylvain Hatier et Rui Yan, «Analyse contrastive des patrons verbaux dans l'écrit scientifique entre scripteurs étudiants et experts », Corela [En ligne], HS-21 | 2017, mis en ligne le 20 mars 2017. consulté le 30 avril 2019. URL : http://journals.openedition.org/corela/4879 ; DOI : 10.4000/ corela.4879

Ce document a été généré automatiquement le 30 avril 2019

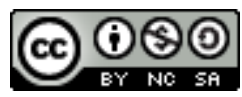

Corela - cognition, représentation, langage est mis à disposition selon les termes de la licence Creative Commons Attribution - Pas d'Utilisation Commerciale - Partage dans les Mêmes Conditions 4.0 International. 


\title{
Analyse contrastive des patrons verbaux dans l'écrit scientifique entre scripteurs étudiants et experts
}

\author{
Sylvain Hatier et Rui Yan
}

\section{Cadre et objectifs de l'étude}

1 Notre étude s'inscrit dans le domaine de la linguistique de corpus, et emprunte à celle-ci le «principe d'idiomaticité » (idiom principle) du fonctionnement de la langue (Sinclair, 1991). Nous appliquons ici ce principe à l'étude d'un lexique spécifique, le lexique scientifique transdisciplinaire (désormais LST), ceci afin de rendre compte des constructions polylexicales récurrentes le concernant. Cette approche étendue de la phraséologie permet de prendre en compte des combinaisons lexico-syntaxiques, dont l'intérêt dans l'enseignement/apprentissage d'une langue étrangère nous paraît essentiel, notamment dans le cadre des langues de spécialité ou de l'« écrit académique ».

Dans le présent article, nous nous intéressons aux patrons verbaux en procédant à une analyse contrastive entre un corpus de mémoires d'étudiants chinois apprenants du français, un corpus de mémoires d'étudiants français en master de didactique du Français et un corpus d'articles de recherche en sciences humaines et sociales parus dans des revues à comités de lecture. Le choix des patrons verbaux dans l'écrit scientifique comme objet d'étude est guidé par des objectifs d'ordre linguistique et didactique. D'une part, à l'instar de Hanks (2013), nous considérons que l'analyse du sens lexical ne peut se faire sur des mots isolés. Les mots ont des sens potentiels, pouvant être activés par les patrons dans lesquels ils s'inscrivent. Ainsi, l'étude des patrons permet de restreindre l'ambiguité $\mathrm{du}$ sens du verbe. D'autre part, comme le souligne Paquot (2010: 118), « les verbes ayant des fonctions rhétoriques ou organisationnelles spécifiques dans l'écrit académique entrent généralement dans des séquences compositionnelles 'flexibles'” ». Or, ce domaine 
lexico-grammatical est une des difficultés majeures rencontrées par les étudiants dans leurs activités rédactionnelles (Cavalla, 2014).

3 À l'instar de Tutin \& Grossmann (2014), nous prenons pour objet d'étude les verbes du lexique scientifique transdisciplinaire. Ce lexique, au cœur de l'argumentation, permet de décrire l'activité scientifique notamment à travers le raisonnement et le positionnement, exprimés par les verbes d'analyse et d'opinion. Le LST est ainsi à la fois méta-scientifique et méta-discursif.

4 Nous avons pour objectif didactique premier l'identification des difficultés liées aux patrons verbaux. Nous dégageons alors les difficultés communes aux étudiants chinois et français ainsi que celles propres aux étudiants chinois. Pour ce faire, nous procédons à une analyse contrastive des corpus d'étudiants natifs/non natifs. À travers l'identification de ces difficultés, nous avons pour perspective l'élaboration d'un outil d'aide à la rédaction scientifique. Nous envisageons ainsi d'intégrer les résultats de l'analyse linguistique des patrons dans des exercices se basant sur la méthode inductive et les corpus pour améliorer l'enseignement/apprentissage des patrons verbaux.

5 Ce travail d'analyse contrastive des patrons verbaux part de plusieurs hypothèses. Premièrement, nous pensons que la variété des patrons verbaux se révèlera moindre dans les corpus d'étudiants que dans le corpus d'experts. Au niveau de la comparaison scripteurs natifs/non-natifs, nous supposons que les étudiants français emploient une plus grande diversité de patrons que les étudiants chinois. L'analyse de ces différences dans l'emploi des patrons verbaux nous permettra également d'en analyser les effets sur les dimensions argumentatives et rhétoriques.

\section{Genres des corpus d'analyse}

6 Nous prenons pour corpus d'analyse différents ensembles de textes se rapportant aux genres de l'écrit scientifique et de littéracie avancée. Le genre étant défini ici, à la suite de Biber (1993), comme une catégorie de textes définis situationnellement. Les deux corpus d'étudiants chinois et français peuvent être regroupés dans un même sous-genre dit de littéracie avancée. Le corpus d'experts appartient lui au sous-genre de l'article de recherche. Lors de l'étape d'analyse contrastive, nous tiendrons compte de ces différences de genre dans les corpus. En effet, comme le souligne Rinck (2013), parmi les multiples facteurs de variation de genre, se trouvent notamment la discipline, l'expertise et le style $\mathrm{du}$ scripteur. Pour l'ensemble de ses genres d'écrit, nous nous intéressons plus précisément au lexique propre à la démonstration scientifique, le LST.

\section{Lexique scientifique transdisciplinaire}

7 Le LST est le lexique spécifique au genre de l'écrit scientifique. Nous le définissons par des propriétés linguistiques et lexicométriques. Ces propriétés nous ont permis, dans de précédents travaux ${ }^{2}$, de procéder à une extraction semi-automatique du LST et d'élaborer une classification sémantique de ce lexique.

\section{Définition}

8 À la suite de Tutin (2007), nous définissons le LST comme le lexique renvoyant au discours sur les objets et les procédures scientifiques. Il est central dans l'argumentation et la 
structuration du discours scientifique, comme le notent notamment Drouin (2007) ou Paquot \& Bestgen (2009).

Du_point de vue anthropologique ce déplacement de paradigme met en jeu la définition même de l'identité en Renfoçant le divorce entre l'identité civile et l'identité personnelle ou sociale ${ }^{4}$

\section{Extraction}

13 À partir d'un corpus d'articles de recherche en sciences humaines et sociales, que nous présenterons en détails dans la partie méthodologie, nous avons procédé à l'extraction d'une liste de mots répondant aux critères de transdisciplinarité et de spécificité. Cette liste a ensuite été validée manuellement par des experts pour aboutir à une ressource du LST composée de 1297 mots pleins (noms, verbes, adjectifs et adverbes).

\section{Classification sémantique}

14 À l'aide des ressources lexicographiques de Dubois \& Dubois-Charlier (1997, 2010), nous avons par la suite enrichi la ressource du LST en informations sémantiques (glose, classes sémantiques). La classification repose sur une typologie à deux niveaux, classes et sousclasses. Celles-ci sont constituées sur la base de propriétés lexico-syntaxiques, à la manière de Flaux \& Van De Velde (2000) et de Dubois \& Dubois-Charlier, suite à l'observation et l'analyse des profils combinatoires ${ }^{5}$ des éléments du LST dans le corpus. Nous disposons ainsi d'une ressource lexicale spécifique au genre de l'écrit scientifique, dont un extrait est présenté dans le tableau ci-dessous.

\begin{tabular}{|l|l|l|l|l|}
\hline Acception & Catégorie & Classe & Sous-classe & Glose \\
\hline article & Nom & $\{$ communication $\}$ & $\{$ document\} & Écrit \\
\hline document & Nom & $\{$ communication $\}$ & $\{$ document $\}$ & Écrit \\
\hline ouvrage & Nom & $\{$ communication & $\{$ document $\}$ & Livre \\
\hline présenter-1 & Verbe & $\{$ état & $\{$ inclusion $\}$ & comporter \\
\hline présenter-2 & Verbe & $\{$ analyse & $\{$ description $\}$ & montrer, exposer \\
\hline
\end{tabular}


Tableau 1 : Exemple d'entrées lexicales du LST

- un corpus d'écrits d'étudiants chinois, de 600000 mots, constitué de mémoires de master ${ }^{8}$ de spécialité de français dans une université chinoise. Leur sujet d'étude porte sur la traduction (9), la littérature (12), la linguistique (1) et des thématiques socioculturelles (7);

- un corpus d'écrits d'étudiants français, de 460000 mots, constitué de mémoires de master en spécialité didactique du français. Ce corpus est issu du corpus Littéracie Avancée ${ }^{9}$ ;

- un corpus d'experts, de 5 millions de mots, issu du projet Scientext ${ }^{10}$, constitué de 500 articles de dix disciplines des sciences humaines et sociales ${ }^{11}$. 


\section{Analyse et enrichissement}

24 dépendances. Les corpus sont ainsi enrichis en lemmes, catégories syntaxiques, traits
morphosyntaxiques et relations syntaxiques. Les traits de classes et sous-classes
sémantiques ont également été projetés dans le corpus afin d'intégrer ces informations
sémantiques dans les patrons verbaux extraits.

\section{Extraction des constructions verbales} décrire, considérer), de fréquence élevée dans les trois corpus et essentiels du point de vue argumentatif, car mobilisés dans le positionnement et analyse scientifique. En effet, les verbes montrer et considérer sont mis à contribution pour construire la «posture réflexive» (Rinck, 2010) de scripteur (ex: nous montrons l'importance, on considère que). Quant aux verbes décrire et expliquer, ils sont employés dans les phases de description et d'interprétation des données, permettant ainsi d'établir des relations entre les éléments linguistiques.

Pour chacun de ces verbes, nous procédons à l'extraction de l'ensemble des occurrences. Chaque occurrence verbale est ensuite représentée par un cadre de sous-catégorisation, extrait automatiquement à partir des résultats de l'analyse en dépendances.

\section{Analyse en dépendance et cadre de sous-catégorisation}

Comme nous l'avons précisé précédemment, les trois corpus (écrits d'étudiants natifs, d'étudiants non-natifs et d'experts) ont été analysés en dépendances avec XIP. Les corpus sont au format XML-TEI. Pour chaque phrase, sont listés l'ensemble des tokens et l'ensemble des relations de dépendances les impliquant dans la phrase. Considérons la phrase suivante :

L'analyse syntaxique et l'enrichissement sémantique nous permettent d'aboutir au résultat suivant :

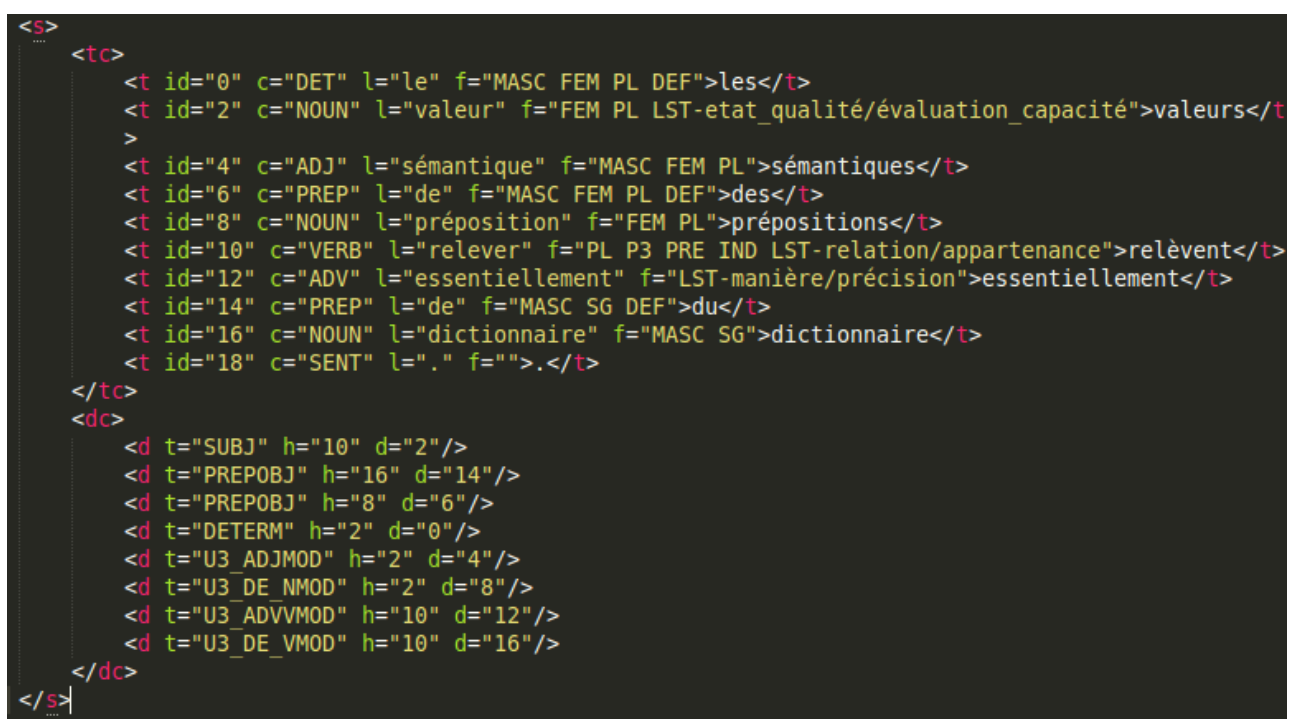


Illustration 1 : Exemple de phrase du corpus au format XML-TEI des cadres de sous-catégorisation «sans apriori, pour faire émerger du corpus [ceux] correspondant à l'usage ». Cette démarche " corpus-driven» (cf. Tognini-Bonelli, 2001), permet de faire émerger à partir du corpus des patrons récurrents. Ces patrons sont obtenus après une phase de factorisation des cadres de sous-catégorisation, à la manière de Kupsc (2007). Nous regroupons ainsi certaines relations de dépendance sous une même étiquette. Par exemple, si le verbe est dépendant dans la relation objet avec un semimodal (devoir, sembler, aller), la relation MODAL-OBJ est ajoutée dans le cadre de souscatégorisation. De plus, les informations sémantiques concernant les cooccurrents du verbe nous permettent de dégager, lorsque cela est possible, des classes ou sous-classes sémantiques préférentiellement associées au verbe pour le cadre en question.

Toute occurrence d'un verbe est alors analysée en termes d'ensemble de relations, tel que l'illustre la figure ci-après :

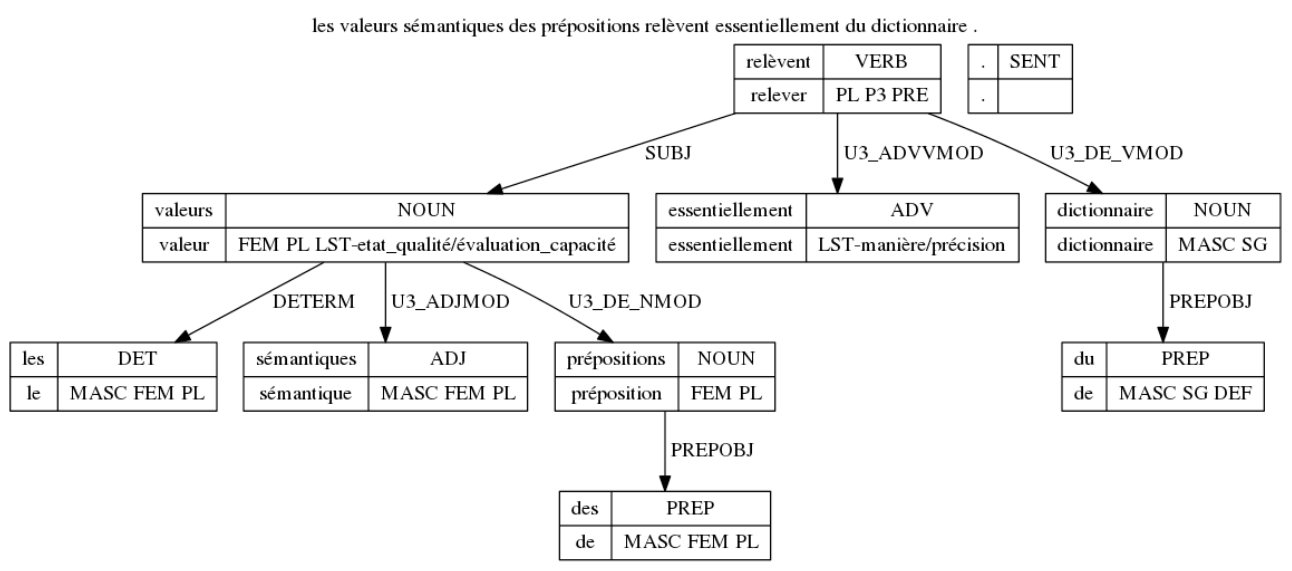

Illustration 2 : Exemple d'analyse en dépendances

Le verbe relever est dans cet exemple :

- gouverneur dans la relation 'sujet'13 avec pour dépendant valeurs (nom du LST, dont la classe et la sous-classe sont renseignées dans les traits);

- gouverneur dans la relation 'adverbiale' avec pour dépendant essentiellement;

- gouverneur dans la relation 'complément prépositionnel' avec pour dépendant dictionnaire.

Étant donné que nous ne retenons pas les compléments adverbiaux dans cette étude, nous obtenons alors le cadre de sous-catégorisation suivant:

(SUBJ \{document\}) relever_VERB (COMPLEMENT PREP.).

Pour chaque cadre, nous calculons les mots en cooccurrence statistiquement significative ${ }^{14}$ avec le verbe concerné. Le même traitement est effectué au niveau des traits sémantiques afin d'identifier les classes intégrant préférentiellement le cadre. Nous intégrons également le trait +hum lorsque le cooccurrent est un des pronoms suivants : je, on, nous.

La phase de factorisation des cadres permet de regrouper au maximum les occurrences et ainsi d'analyser les structures les plus fréquentes. Nous obtenons pour le verbe montrer, dans le corpus d'experts, les cadres présentés ci-dessous. 


\begin{tabular}{|c|c|c|}
\hline Cadre & Occurrences & Exemples \\
\hline (SUBJ-hum)montrer_VERB(COMPLETIVE_QUE) & 762 & $\begin{array}{l}\text { Texte_psycho_32_XipEmolex.xml : Les résultats } \\
\text { montrent que le facteur qui influence plus } \\
\text { particulièrement la performance des enfants est I' } \\
\text { alignement de la position finale avec un axe implicite } \\
\text { du dispositif ( vertical ou horizontal). }\end{array}$ \\
\hline (SUBJ -hum)montrer_VERB(OBJ -hum) & 275 & $\begin{array}{l}\text { Texte_psycho_8_XipEmolex.xml : Les résultats } \\
\text { montrent un effet signifi-catif de la présence de l' } \\
\text { appareil , } t(24)=-2,27 ; p \text { \&lt; } 0,03 \text {. }\end{array}$ \\
\hline (SUBJ +hum)montrer_VERB(COMPLETIVE_QUE) & 150 & $\begin{array}{l}\text { Texte_economie_10_XipEmolex.xml : Nous } \\
\text { montrons qu' il n' en est rien. }\end{array}$ \\
\hline
\end{tabular}

Illustration 3 : Cadres de sous-catégorisation du verbe montrer

nous est alors possible d'identifier le cadre le plus fréquent (ici 762 occurrences) pour le verbe montrer, comprenant un sujet non humain et une complétive introduite par que. Sont également renseignés, pour chaque cadre, des exemples, ainsi que les mots et classes les plus fréquents. Les résultats de cette phase d'extraction et de factorisation des cadres de sous-catégorisation intègrent ainsi des informations d'ordres syntaxique, lexical et sémantique. Ces informations sont alors utilisées dans la seconde phase de regroupement manuel présentée dans la partie suivante.

\section{Patrons lexico-syntaxiques et modèle Corpus Pattern Analysis}

Le modèle Corpus Pattern Analysis (CPA), du domaine de l'analyse lexicale, envisage le sens en contexte, inscrit dans les patterns. Cette approche est dans la continuité de la linguistique de corpus de Sinclair dans la mesure où les patterns ne sont repérables que par le biais d'analyses de fréquence inductives (corpus driven approach). Elle se distingue des travaux précédents sur les patterns, notamment l'approche de Pattern Grammar (Hunston \& Francis, 2000) en accordant un rôle clé aux collocations lexicales pour déterminer la notion de pattern :

A pattern is a statement of the clause structure (valency) associated with a meaning of a verb, together with typical semantic values of each argument, realized by salient collocates [...] Different semantic values of arguments activate different meanings of each verb ${ }^{15}$. (Hanks, 2013)

Ce modèle est d'abord utilisé dans la construction du Pattern Dictionary of English Verbs $\left(\mathrm{PDEV}^{16}\right)$ pour l'anglais langue générale (Hanks, 2013). Nous illustrons ci-dessous un exemple de pattern dans le PDEV :

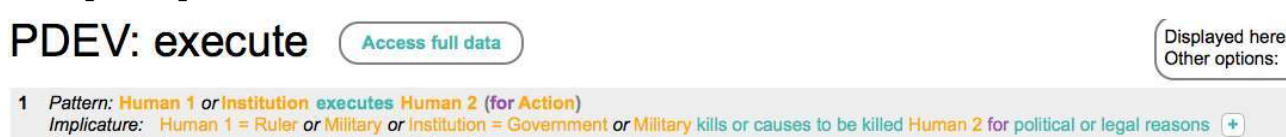

Illustration

Dans cet exemple du verbe to execute, nous pouvons voir que le pattern définit pour chacun de ses arguments des "types sémantiques" ou concepts cognitifs tels que " human ", «institution», "action » etc. Les types sémantiques peuvent être complétés par des « rôles sémantiques » en vue d'affiner la définition du pattern. Ces rôles représentent 
les propriétés extrinsèques des mots au niveau de chaque argument, en fonction du contexte du pattern. Dans l'exemple ci-dessus, la séquence "human $1=$ Ruler or Military " signifie que le type sémantique "human" s'instancie dans le rôle du souverain ou du militaire dans ce contexte. L'implicature renvoie ainsi à une paraphrase du pattern.

Plusieurs projets en cours montrent que le modèle CPA est adapté à des objectifs divers :

- étude lexicale dans un domaine spécifique comme l'écrit académique - l'E-Advanced Learner's Dictionary of Verbs in Science (DicSci) (Williams, Million \& Alonso, 2012);

- étude quantitative des structures verbes arguments en espagnol (Nazar \& Renau, à paraître) ;

- étude des verbes espagnols à visée pédagogique pour le Diccionario de aprendizaje del español como lengua extranjera (DAELE ${ }^{17}$ ) (Renau \& Battaner, 2011).

Concernant notre étude, nous appliquons le modèle CPA à l'écrit scientifique en français dans une perspective didactique. L'identification des difficultés sur les constructions verbales dans les écrits scientifiques constitue la première étape dans cet objectif. En effet, ce diagnostic nous amène à poser des hypothèses explicatives qui nous guident dans notre réflexion didactique afin d'aider les étudiants (non-natifs) dans la rédaction scientifique.

Les cadres de sous-catégorisation sont ensuite analysés manuellement et regroupés dans des patrons proches du modèle CPA, incluant des informations lexicales, syntaxiques et sémantiques. Ainsi, si nous prenons pour exemple le verbe expliquer, considérons un de ses cadres de sous-catégorisation :

- (SUBJ-hum) (SE se_PRON) expliquer_VERB par (92 occurences).

- Ex 1: La différence s'explique sans doute par la proximité de ces élus avec leurs administrés, souvent plus favorables à Bonn. (corpus experts : géographie)

Ce cadre décrit un emploi pronominal du verbe expliquer. Dans ce cadre, le trait -hum du sujet renvoie à un nom non humain dont les plus fréquents sont différence, situation, résultat, fait. Les types sémantiques utilisés préférentiellement comme sujets dans ce cadre sont la classe \{relation\}, avec le nom différence, et la sous-classe \{situation\} de la classe \{objet scientifique\}, avec les noms situation et fait comme illustré dans l'exemple 2 :

- Ex 2 : Le fait que les corrélations avec l'absentéisme soient sensiblement plus élevées au niveau des unités de soins qu'au niveau individuel peut s'expliquer par le fait que la variable absentéisme reflète la seule variance entre les unités.

À ces classes s'ajoutent des noms renvoyant à la classe \{processus cognitifs\}, tel choix. Ces occurrences correspondent aux cas dans lesquels l'auteur se justifie, se positionne, comme l'illustre l'exemple 3 :

- Ex 3: Ce choix s'explique par le fait qu'elles sont particulièrement appropriées aux échantillons composés de personnes qui occupent un emploi commercial comme dans le cas de notre échantillon. (corpus experts : psycho)

En identifiant ainsi les classes sémantiques en cooccurrence significative avec le verbe, nous pouvons intégrer cette dimension sémantique dans les patrons. Cependant, le recouvrement entre classes sémantiques et noms rentrant effectivement en cooccurrence avec le verbe est évidemment partiel. Une classe sémantique peut être fréquemment en relation avec le verbe à travers une minorité de ses éléments tandis que ses autres éléments n'entrent jamais en cooccurrence avec le même verbe. L'objectif n'est pas de relever l'intégralité des combinaisons lexicales mais d'intégrer des informations sémantiques afin de décrire le plus finement possible les patrons. 
Si nous reprenons l'exemple du cadre pronominal du verbe expliquer, nous voyons qu'il est suivi de la préposition par, qui introduit l'objet de la cause. Cet élément est fréquemment réalisé par un nom de la classe \{qualité\} (nécessité, difficulté, propriété) et de la classe \{objet scientifique\} (phénomène, méthode). Nous pouvons donc présenter le patron lexico-syntaxique comme suit :

Patron: [[relation|objet scientifique|processus cognitif]] s'explique par [[qualité|objet scientifique]]

Implicature : [[qualité|objet scientifique]] constitue la raison de [[relation|objet scientifique|processus cognitif]] et le rend compréhensible. choix, situation, résultat, problème

À la suite de cette étape d'analyse des cadres de sous-catégorisation et de création des patrons, nous pouvons procéder à l'étude contrastive des constructions verbales entre les trois corpus.

\section{Étude contrastive des constructions verbales}

51 Le corpus d'experts nous servira ici de référence pour identifier les patrons les plus fréquents dans l'écrit scientifique. Nous nous appuierons sur cette base pour confronter les deux corpus d'étudiants, comparables au niveau de la taille et du contexte d'écriture (mémoire de fin d'études).

\section{Fréquences et analyse quantitative}

52 Avant de nous intéresser plus spécifiquement à quatre verbes du LST (décrire, expliquer, montrer, considérer) et à leurs constructions, nous présentons dans cette partie les points communs et spécificités des corpus pour l'ensemble des verbes du LST. Ce lexique, constitué à partir du corpus d'experts, est composé de 342 verbes, dont 334 sont présents dans le corpus d'étudiants chinois et 336 dans le corpus d'étudiants français. Cela rejoint notre remarque précédente selon laquelle ce lexique est central dans le genre de l'écrit scientifique et essentiel à maîtriser pour les étudiants.

53 Si nous nous intéressons aux verbes les plus fréquents, nous constatons que parmi les 50 verbes les plus présents dans chaque corpus d'étudiants, 28 sont en commun: devenir, exister, utiliser, exprimer, présenter, considérer, constituer, montrer, décrire, développer, analyser, concerner, sembler, apparaître, permettre, produire, choisir, expliquer, représenter, réaliser, établir, proposer, étudier, paraitre, apporter.

Les corpus d'étudiants diffèrent cependant sur la fréquence de certains verbes. Par exemple, le verbe semi-modal permettre est le plus fréquent chez les étudiants français (fréquence par million de mots de 2982 contre 294 pour le corpus d'étudiants chinois). Il est ainsi largement utilisé dans des constructions où un \{processus_cognitif\} ou un \{objet scientifique\} précède 'permettre $d e$ ', une construction essentielle dans l'argumentation scientifique (voir exemples 4 et 5 ).

55 - Ex 4: Les exemples ci-dessus nous permettent de tirer la conclusion: la qualité de traduction littérale est qu'elle conserve le plus possible l'image [...] (corpus d'étudiants chinois) 
56 - Ex 5 : Cette prise de conscience du sens de l'écriture par la production d'écrit, permet d'impliquer d'avantage les élèves dans leur apprentissage [...] (corpus d'étudiants français)

57 À l'inverse, parmi les verbes fréquents dans le corpus d'étudiants chinois mais absents ou peu fréquents dans le corpus d'étudiants français, nous identifions les verbes suivants: traduire, obtenir, former, signifier, influencer, posséder, indiquer, composer, manifester, mentionner, publier, correspondre, marquer, atteindre, refléter, conduire, construire, exercer.

Le verbe traduire (fréquence par million de mots de 1083 contre 53 pour le corpus d'étudiants français) est le plus fréquent chez les étudiants chinois. Ceci n'est cependant pas dû à l'emploi de l'acception scientifique ('être l'expression d'un phénomène'), mais à son acception appartenant à la langue générale 'formuler dans une autre langue étrangère', liée à la thématique du corpus d'étudiants chinois, la traduction. Nous faisons le même constat pour d'autres verbes (obtenir place/permission, manifester sentiment) pour lesquels l'acception dans le corpus ne correspond à aucune entrée lexicale de notre ressource du LST. Ainsi, certains de ces verbes ne sont pas représentatifs de ce lexique spécifiquement mobilisé dans les écrits scientifiques. De plus, d'autres verbes, bien qu'éléments du LST, sont employés dans un sens ne s'inscrivant pas dans notre définition du LST.

59 Par exemple, si nous comparons les exemples suivants :

- Ex 6: Pas mal de chercheurs traduisent leurs propres essais académiques en une autre langue pour les publier dans les magazines ou journaux étrangers [...]. (corpus d'étudiants chinois)

- Ex 7: L'inacceptabilité de contre dans ces contextes traduit là encore le fait que l'individu visé par ces sentiments n'est supposé exercer aucune contre-force. (corpus experts : linguistique)

60 Nous constatons dans l'exemple 6 un emploi non-LST du verbe traduire dont la haute fréquence dans le corpus d'étudiants chinois est bien causée par la thématique des écrits. De la même manière, la thématique du corpus d'étudiants français étant la didactique, nous y trouvons parmi les verbes les plus fréquents écrire et lire. L'analyse qualitative des concordances est ici nécessaire pour savoir si les verbes sont effectivement utilisés dans leur sens scientifique ou si les étudiants (français et/ou chinois) ne maîtrisent pas l'emploi de ce sens spécialisé.

61 Concernant les verbes surreprésentés dans le corpus d'étudiants français, en comparaison du corpus d'étudiants chinois, nous identifions : observer, impliquer, effectuer, intéresser, adapter, favoriser, constater, souligner, définir, correspondre, amener, aborder, participer, interroger, noter, préciser, reconnaître, faciliter, évaluer, progresser, identifier, appuyer.

Ces verbes sont également fréquemment employés dans la description de l'activité scientifique dans le corpus d'experts. Ils appartiennent notamment aux classes sémantiques suivantes : \{constat\} (observer, constater, noter), \{opinion\} (souligner), \{analyse\} ( définir, préciser).

63 Nous notons ainsi une certaine convergence concernant les verbes du LST employés par les étudiants chinois et français. Cependant, les différences de fréquence sont un indice de la variabilité de l'emploi de ces verbes, et notamment de leur usage spécifique dans l'écrit scientifique. Afin d'étudier ces possibles divergences, nous procédons à une analyse qualitative des patrons présents dans les différents corpus, et ce, pour quatre verbes du LST. 


\section{Analyse qualitative avec l'outil Anatext}

Nous précisons ici que notre méthodologie d'extraction semi-automatique des patrons a dans un premier temps été élaborée pour le corpus d'experts dont la taille est dix fois supérieure à celle des corpus d'étudiants. Les résultats de l'étape de factorisation des cadres (reposant sur des effectifs nombreux) sont alors insuffisants pour une analyse basée uniquement sur les calculs de fréquence. Ce changement d'échelle explique le recours à un outil supplémentaire pour procéder à l'analyse qualitative des patrons, et notamment à l'étude des classes sémantiques s'inscrivant dans les patrons. Nous utilisons ainsi, pour l'étape d'observation du corpus, l'outil de textométrie Anatext ${ }^{18}$, conçu par Olivier Kraif.

Les quatre verbes du LST (montrer, considérer, décrire et expliquer) que nous étudions spécifiquement sont parmi les plus fréquents pour chaque corpus analysé, ce qui permet d'extraire et d'observer le maximum d'occurrences. Le tableau suivant présente leur fréquence par million de mots (et fréquence absolue entre parenthèses) dans chaque corpus :

\begin{tabular}{|l|l|l|l|}
\hline & Experts & Étudiants français & Étudiants chinois \\
\hline montrer & $649(3114)$ & $780(363)$ & $449(271)$ \\
\hline considérer & $533(2259)$ & $367(171)$ & $469(283)$ \\
\hline décrire & $236(1134)$ & $221(103)$ & $384(232)$ \\
\hline expliquer & $341(1637)$ & $896(417)$ & $265(160)$ \\
\hline
\end{tabular}

Tableau 2 : Fréquences des quatre verbes dans les trois corpus

Comme nous pouvons le constater, les verbes montrer et considérer sont les plus fréquemment utilisés dans les trois corpus. Le verbe décrire est surreprésenté dans le corpus d'étudiants chinois, le verbe expliquer dans le corpus d'étudiants français. Nous posons l'hypothèse que ces variations ont deux facteurs principaux: d'une part, la thématique des mémoires (didactique de l'écrit pour les étudiants français et traduction/ littérature pour les étudiants chinois), et d'autre part la compétence discursive des deux groupes de scripteurs>

Nous présentons dans la partie suivante l'analyse contrastive des patrons grammaticaux, en introduisant tout d'abord les patrons lexico-syntaxiques répertoriés en se basant sur le corpus d'experts. Les patrons grammaticaux relèvent de l'approche « Pattern Grammar » et n'incluent pas les collocations. Dans un premier temps, afin de faciliter la comparaison et pour avoir une vision générale des constructions, l'analyse des patrons dans les corpus d'étudiants se fera au niveau des patrons grammaticaux. Ces patrons grammaticaux résultent de l'extraction des cadres de sous-catégorisation. Les patrons lexicosyntaxiques se situent à un niveau d'analyse supérieure, nécessitant un traitement manuel des arguments verbaux récurrents, uniquement effectué sur le corpus d'expert. 
La prise en compte des phénomènes collocatifs sera effective pour l'étape d'analyse suivante, qui prend notamment appui sur les calculs de cooccurrence.

\section{Verbe montrer}

Afin de ne pas devoir traiter un trop grand nombre de cadres de sous-catégorisation différents, nous fixons le seuil de fréquence minimale par cadre à 15 pour le corpus d'experts et à 5 pour les corpus d'étudiants.

Les patrons lexico-syntaxiques extraits du corpus d'experts sont filtrés selon le critère de transdisciplinarité. Ce corpus étant composé d'articles de 10 disciplines, nous ne retenons que les cadres non spécifiques à une seule discipline. Ce critère de transversalité est également appliqué pour les compléments nominaux appartenant au LST intégrant ces cadres lexico-syntaxiques.

Nous présentons ci-dessous un exemple des patrons lexico-syntaxiques du verbe montrer. Le formalisme, intégrant informations syntaxiques, lexicales et sémantiques, est inspiré du modèle CPA.

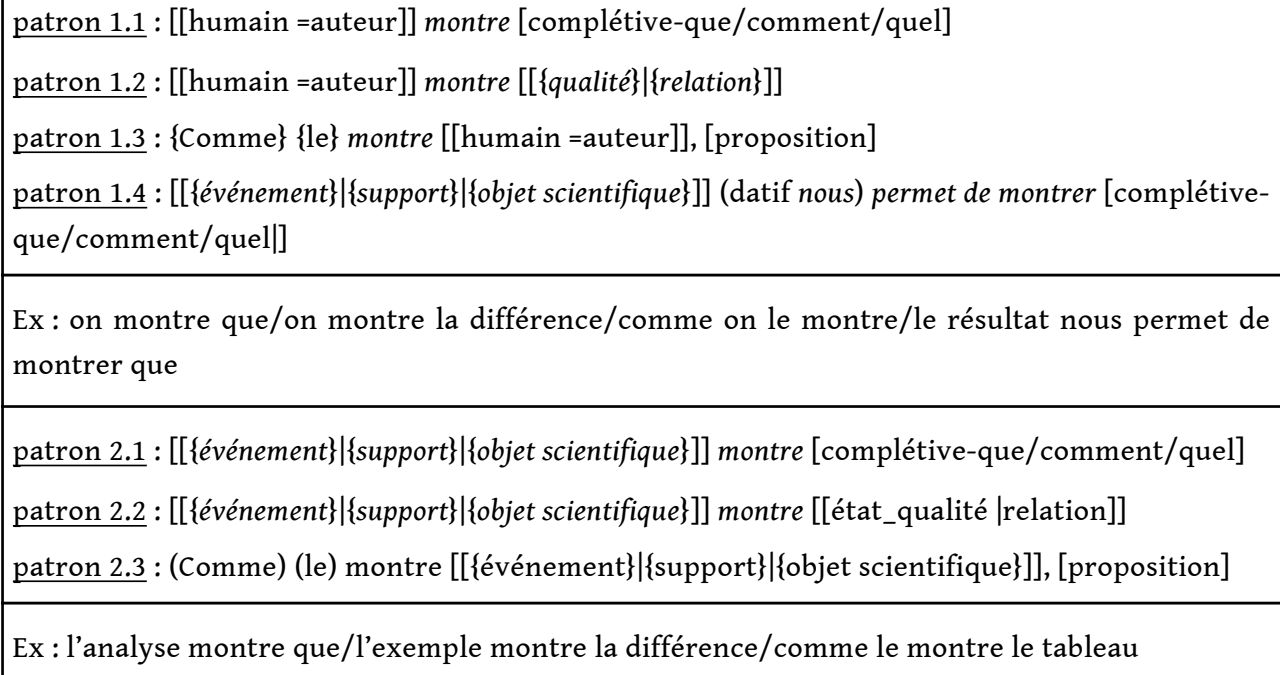

Tableau 3 : Patrons lexico-syntaxiques du verbe montrer dans le corpus d'experts

71 Dans les patrons pour lesquels le verbe sélectionne un sujet humain, montrer prend le sens de 'donner des arguments pour faire savoir et justifier certains faits scientifiques'. Lorsque montrer sélectionne un sujet de type chose, le verbe prend le sens 'mettre en évidence'.

73 La modélisation des patrons permet ainsi d'associer des constructions lexico-syntaxiques à des acceptions identifiées.

74 Nous confrontons maintenant ces patrons, issus du corpus d'experts, aux patrons grammaticaux du verbe montrer dans les deux corpus d'étudiants. Le tableau ci-dessous présente les rangs de fréquence pour les patrons du verbe montrer dans l'ensemble des corpus. Un rang 1 signifie que le patron est le plus utilisé pour le verbe en question dans le corpus. La valeur'-' signifie que le patron est absent du corpus, ou que sa fréquence est inférieure au seuil de 5 occurrences. 


\begin{tabular}{|l|l|l|l|}
\hline Patrons grammaticaux & Experts & Français & Chinois \\
\hline (SUBJ chose) montrer que & 1 & 2 & 3 \\
\hline (SUBJ humain) montrer que & 2 & 1 & 3 \\
\hline (SUBJ humain) montrer (OBJ chose) & 3 & 3 & 2 \\
\hline (SUBJ chose) montrer (OBJ chose) & 3 & 3 & 1 \\
\hline Comme le montrer (SUBJ humain) & 4 & - & - \\
\hline Comme le montrer (SUBJ chose) & 4 & - & - \\
\hline (SUBJ chose) permet de montrer que & 4 & 4 & - \\
\hline
\end{tabular}

Tableau 4 : Ordre des fréquences des patrons du verbe montrer dans les trois corpus

Ce tableau nous révèle que les étudiants chinois ont plus de tendance à utiliser des patrons intégrant un complément d'objet nominal chose tandis que les experts et les étudiants français emploient plus fréquemment la structure en complétive que. Parmi les patrons avec complétive, celui prenant un sujet humain est fréquemment employé par les experts et étudiants français. Cependant, l'observation des concordances fait apparaître une divergence dans l'emploi de ce patron. Ce dernier est utilisé notamment pour marquer le positionnement, associé aux pronoms personnels on ou nous dans le corpus d'experts. Cependant, ce positionnement n'est pas directement «assumé » par les scripteurs étudiants, qui renvoient alors souvent à un autre chercheur du domaine pour soutenir l'argumentation. Le recours au discours rapporté ne marque alors pas une opinion explicite et le point de vue de l'auteur est dissimulé en faisant dire par autrui ce qu'il souhaiterait exprimer. L'argumentation se construit ainsi comme dans l'exemple suivant:

- Ex 8 : Nadeau et Fisher (2011), montrent que les connaissances explicites sont corrélées au degré de certitude des apprenants concernant leurs réponses. (corpus d'étudiants français).

Le même constat est fait pour les étudiants chinois qui emploient très peu les pronoms on ou nous mais plutôt des renvois :

- Ex 9: De plus, la conclusion de XU Minglong montre que la «sinomanie» est un phénomène important dans l'histoire des échanges culturels entre la Chine et l'Europe. (corpus d'étudiants chinois).

77 Cependant, les patrons les plus fréquemment employés par les étudiants chinois activent le sens 'faire voir' du verbe montrer, comme dans l'exemple 10 :

- Ex 10: Le tableau en bas peut montrer les diplômes différents dans les étapes différentes. (corpus d'étudiants chinois).

78 Si nous considérons les autres patrons présents dans le corpus d'experts, nous constatons que les étudiants emploient peu de constructions infinitives. Ainsi, les patrons incluant les verbes suggérer, nécessiter, permettre et les constructions en comme sont peu utilisées 
par les étudiants. Pourtant, il nous semble que ces constructions sont essentielles dans l'argumentation (ces résultats permettent de montrer) et dans la structure du texte (comme on l'a montré). Les étudiants semblent ainsi avoir des difficultés à exprimer leurs propres opinions et à faire des renvois textuels.

\section{Verbe considérer}

L'analyse qualitative des patrons du verbe considérer fait émerger des différences comparables à l'exemple du verbe montrer.

Nous présentons ci-dessous les patrons modélisés à la suite des traitements automatiques et de l'observation du corpus d'experts :

\begin{tabular}{|c|}
\hline Ex : on considère la relation comme \\
\hline patron 2: [[humain =auteur]] considère [complétive-que] \\
\hline Ex : on considère que \\
\hline $\begin{array}{l}\text { Patron 3 } \\
\text { [proposition] }\end{array}$ \\
\hline
\end{tabular}

Tableau 5 : Patrons lexico-syntaxiques du verbe considérer dans le corpus d'experts

81 Ce verbe correspond à trois entrées lexicales, ou acceptions, dans notre ressource du LST : 'donner un jugement', 'exprimer une opinion' et 'prendre en considération'.

Le patron 3, recouvrant le dernier sens, est notamment utilisé lorsque l'auteur introduit un nouvel argument, la [proposition]. Cet argument est alors en corrélation avec le nom objet de considérer, représenté par: [[\{objet scientifique\}|\{qualité\}=sujet]]. Ainsi, dans l'exemple suivant, le nom pertinence, de la classe du LST \{qualité\} permet d'introduire la [proposition] qui suit :

- Ex 11 : Si l'on considère, maintenant, les pertinences évoquées par l'orateur, elles sont de deux ordres : la pertinence coranique, tout d'abord, puisqu'il affirme que le projet de loi a été examiné par une assemblée d'experts religieux... (corpus experts : sociologie) L'emploi de ce patron est ici justifié par sa fonction méta-discursive.

Le tableau ci-après présente les fréquences des patrons du verbe considérer dans les différents corpus,

\begin{tabular}{|l|l|l|l|}
\hline Patrons grammaticaux & Experts & Français & Chinois \\
\hline (SUBJ humain) considérer que & 2 & 2 & 2 \\
\hline (SUBJ humain) considérer (OBJ chose) comme & 3 & 1 & 1 \\
\hline
\end{tabular}




\begin{tabular}{|l|l|l|l|}
\hline$(\mathrm{Si})$ considérer (OBJ chose) & 1 & - & - \\
\hline
\end{tabular}

Tableau 6 : Ordre des fréquences des patrons du verbe considérer dans les trois corpus permis d'identifier un seul sens pour le verbe décrire :'représenter en détail'. Ce verbe est régulièrement utilisé au passif comme dans l'exemple suivant :

- Ex 12: Les indicateurs de ségrégation ont été calculés selon une méthode décrite par Stephen Gorard et Chris Taylor (2002). (corpus experts : sciences de l'éducation)

patron $1:[[$ humain $=$ auteur $\mid\{$ processus\} $\}]]$ décrit $[[$ processus\} $\mid\{$ objet scientifique $\}]]$ (comme)
Ex : nous décrivons les effets
souvent au passif : effets décrits

Tableau 7 : Patron lexico-syntaxique du verbe décrire dans le corpus d'experts

Ce patron sélectionne un sujet humain ou un sujet métonymique du type étude, travaux, renvoyant à un humain :

- Ex 13 : Des travaux en sciences de l'éducation, étudiant les pratiques enseignantes [...] décrivent, de façon très précise, le comportement de l'enseignant. (corpus experts: sciences de l'éducation)

Nous observons cependant que cet emploi métonymique est peu fréquent chez les étudiants, en particulier chez les étudiants chinois. 
89 L'analyse des concordances nous révèle également que les experts privilégient la construction au passif réduit, à l'inverse des étudiants, comme on peut le constater dans le tableau suivant :

\begin{tabular}{|l|l|l|l|}
\hline Patrons grammaticaux & Experts & Français & Chinois \\
\hline (OBJ chose) être décrit (passif) & 1 & 2 & 2 \\
\hline (SUBJ humain) décrire (OBJ chose) & 2 & 1 & 1 \\
\hline
\end{tabular}

Tableau 8 : ordre des fréquences des patrons du verbe décrire dans les trois corpus

Les deux exemples suivants illustrent cet emploi du verbe décrire dans les corpus d'étudiants :

- Ex 14: Et SENORE (2011; p60) décrit «la tâche» de cet enseignement comme conduisant « les élèves vers le pouvoir d'écrire ». (corpus d'étudiants français)

- Ex 15 : Dans le livre écrit par l'Abée ${ }^{19}$ Gainet que nous avons mentionné dessus, il a décrit les conditions de vie des Chinois ordinaires vues et racontées par un missionnaire français en Chine. (corpus d'étudiants chinois)

91 Notons par ailleurs que la construction il décrit + objet chose est assez récurrente chez les étudiants chinois. Les noms objets de décrire sont variés et appartiennent rarement au LST : image, scène, condition. Le verbe décrire est ainsi peu utilisé dans la description des données chez les étudiants.

\section{Verbe expliquer}

Concernant le verbe expliquer, l'analyse des cadres et la modélisation des patrons fait ressortir cinq constructions fréquentes dans le corpus d'experts. Ces patrons correspondent aux deux sens 'faire comprendre' et 'justifier et être la cause de'. Ces deux sens se distinguent par la nature de leur sujet, humain ou chose, comme illustré dans le tableau ci-après.

\begin{tabular}{|c|}
\hline Ex : on explique le fait \\
\hline Patron $2.1:[[\{$ qualité $\} \mid\{$ relation $\}=$ cause $]]$ explique $[[\{e ́ v e ́ n e m e n t\}=$ conséquence $]]$ \\
\hline patron 2.2: $[[\{$ qualité\} $\mid\{$ relation $\}=$ cause $]]$ explique $[$ complétive-que/pourquoi/comment $]$ \\
\hline patron 2.3: $:[[$ événement $\}=$ conséquence $]]$ s'explique par $[[\{$ qualité $\} \mid\{$ relation $\}=$ cause $]]$ \\
\hline
\end{tabular}


Tableau 9 : Patrons lexico-syntaxiques du verbe expliquer dans le corpus d'experts L'analyse contrastive des trois corpus pour les
distincts, résumés dans le tableau ci-dessous.

\begin{tabular}{|l|l|l|l|}
\hline Patrons grammaticaux & Experts & Français & Chinois \\
\hline (SUBJ chose) expliquer (OBJ chose) & 1 & 3 & 3 \\
\hline (OBJ chose) s'expliquer par & 2 & - & - \\
\hline (OBJ chose) expliquer que & 3 & - & - \\
\hline (SUBJ humain) expliquer que & - & 1 & 2 \\
\hline (SUBJ humain) expliquer (OBJ chose) & - & 2 & 1 \\
\hline
\end{tabular}

Tableau 10 : ordre des fréquences des patrons du verbe expliquer dans les trois corpus syntaxiques : le passif et le pronominal de sens passif. De la même manière que pour les
patrons du verbe montrer, les étudiants français favorisent les patrons avec complétive
lorsque les étudiants chinois privilégient un complément d'objet nominal. 
sous-utilisation :

- de certaines alternances syntaxiques : passif, passif pronominal ;

- d'éléments lexicaux : mots du LST, pronom sujet on et nous ;

- de certaines constructions spécifiques : comme nous verbe, si nous verbe.

À l'inverse, les écrits étudiants, en comparaison des écrits d'experts, sont caractérisés par une sur-utilisation du pronom il et de noms appartenant à la langue générale. Ainsi, les étudiants, français et chinois, ont des difficultés à maîtriser certaines constructions spécifiques à l'écrit scientifique, permettant de développer l'argumentation, d'organiser le texte. Ceci peut alors se traduire par des difficultés à établir une structure discursive cohérente, construire des opinions au sujet des savoirs d'une discipline. Ces difficultés sont encore plus grandes pour les étudiants chinois, du fait de leur statut d'apprenant du français, et aboutissent à des confusions de registre, erreurs de collocations, expressions/ tournures maladroites.

\section{Réflexions pour l'enseignement/apprentissage des patrons verbaux en classe du FLE}

Afin de remédier aux difficultés des étudiants relevées par l'étude contrastive, nous proposons un enseignement des patrons verbaux, en classe de FLE, basé sur corpus et privilégiant l'approche inductive.

103 L'utilisation du corpus dans l'apprentissage répond aux principes $\mathrm{du}$ Data-Driven Learning (DDL) (Johns, 1991) et à l'instar de Chambers et al. (2011) et de Cavalla \& Loiseau (2014), nous pensons que l'enseignement/apprentissage du lexique peut être facilité par la mise en contexte et l'analyse des concordances, tant dans la phase de la découverte des patrons que dans la phase de production écrite.

104 L'approche inductive est largement utilisée dans l'enseignement de la grammaire (Chartrand, 1996), du lexique (Cavalla, 2014) et dans l'acquisition langagière (Colletta, 2004). Cette approche vise à construire l'autonomie et la "posture réflexive" pour l'apprenant (Rinck, 2010 ; Boch \& Buson, 2012).

105 L'étape de transposition didactique des patrons verbaux doit suivre une progression allant de la découverte à la réutilisation en passant par le renforcement et l'approfondissement des connaissances pour l'apprenant. De plus, l'utilisation du corpus doit être guidée par l'enseignant pour éviter de confronter les apprenants à des exemples trop nombreux et/ou complexes.

Nous présentons dans le tableau 11 un exemple de concordances (extraites du corpus d'experts) du verbe considérer. Ces concordances permettent d'appuyer l'apprentissage sur des emplois attestés, en contexte.

\begin{tabular}{|l|l|l|}
\hline $\begin{array}{l}\text { 1. Dans la préface à l'édition des } \\
\text { lettres de Jean Hus de } 1537 \text {, Luther }\end{array}$ & considère & $\begin{array}{l}\text { le réformateur de la Bohême comme un } \\
\text { «vrai chrétien », qui a produit de bons } \\
\text { fruits . }\end{array}$ \\
\hline $\begin{array}{l}\text { 2. Puisque la nature ordinaire } \\
\text { regroupe les milieux et espèces les } \\
\text { plus communs et abondants, on } \\
\text { peut }\end{array}$ & considérer & $\begin{array}{l}\text { que c' est bien celle-ci qui fournit le plus de } \\
\text { ressources à l' humanité . }\end{array}$ \\
\hline
\end{tabular}




\begin{tabular}{|l|l|l|}
\hline 3. Il convient néanmoins de & considérer & $\begin{array}{l}\text { le rôle didactique du théâtre et le contexte } \\
\text { mouvant des années soixante avant de } \\
\text { conclure à une provocation outrancière }\end{array}$ \\
\hline 4. Deux points sont à & considérer & $\begin{array}{l}\text { : d'une part , le sens qu' exprime une } \\
\text { préposition sous les différentes formes qu' } \\
\text { elle est capable de prendre, d'autre part... }\end{array}$ \\
\hline $\begin{array}{l}\text { 5. Et de l' autre, un échange tellement } \\
\text { indirect qu' on peine à voir comment le } \\
\text { principe de réciprocité est respecté. }\end{array}$ & Considérons & $\begin{array}{l}\text { tout d'abord la première forme d'échange, qui } \\
\text { patrilatérale, }\end{array}$ \\
\hline
\end{tabular}

Tableau 11 : concordances du verbe considérer

107 L'apprenant peut ainsi observer les différents aspects des constructions, guidé par les exemples validés par l'enseignant : associations lexicales, alternances syntaxiques (passif, impératif), acception mobilisée, fonction discursive (topicalisation, discours rapporté), constructions spécifiques (il convient de considérer, on peut considérer), etc.

108 Les exemples facilitent ainsi la compréhension et l'appropriation des patrons associés à un sens donné du verbe, et autorisent par exemple la mise en place d'exercices de reformulation, de mise en correspondance de verbes synonymes, pour guider l'apprentissage des constructions verbales.

109 En associant l'observation des concordances à l'étude des patrons, l'enseignant peut ainsi orienter l'apprenant dans la compréhension et l'appropriation de ces constructions complexes.

\section{Perspectives et conclusion}

110 L'extraction semi-automatique des patrons lexico-syntaxiques des verbes du LST nous a permis d'identifier les constructions les plus fréquentes pour plusieurs types de scripteurs. L'analyse contrastive des patrons a mis en évidence les spécificités des étudiants par rapport aux experts et les points de convergence et de divergence entre écrits d'étudiants français et chinois. Notre hypothèse d'une variété des constructions supérieure dans le corpus d'experts a pu être confirmée par les résultats. Certaines différences doivent cependant être confrontées à l'hétérogénéité des corpus. Nous avons en effet pu constater que les sous-genres de l'article de recherche et du mémoire ne sont pas entièrement comparables, notamment au niveau de la structure textuelle. De plus, la thématique des écrits (spécifiquement pour les mémoires) a été identifiée comme facteur de variabilité important dans l'emploi des patrons verbaux et dans les acceptions mobilisées pour chaque verbe étudié. Ainsi, le patron «l'enseignant explique que » est surreprésenté dans les mémoires de didactique de l'écrit lorsque les constructions du type «l'écrivain décrit la scène » sont surreprésentées dans les mémoires en littérature/ traduction.

111 L'analyse contrastive montre également que les difficultés liées aux fonctions discursives sont largement partagées par les étudiants natifs et non-natifs, contrairement à notre intuition de départ. La variété des constructions syntaxiques est ainsi moindre pour les 
étudiants par rapport au corpus d'experts, notamment pour les constructions syntaxiques typiques de l'écrit scientifique, ayant une fonction argumentative ou d'organisation textuelle. Une différence importante entre scripteurs étudiants et experts se situe au niveau lexical des patrons. Ainsi, les compléments sujets des patrons d'écrits étudiants sont peu marqués par la présence de l'auteur, ce qui peut dénoter une dimension énonciative peu présente et un rejet de positionnement. Nous avons également pu observer certaines variations entre le corpus d'étudiants chinois et français, avec par exemple la différence marquée entre l'emploi de compléments d'objet (généralement des noms de la langue générale pour les premiers versus des complétives pour les seconds).

La non-maîtrise de certains patrons dans les corpus d'étudiants est combinée à une surreprésentation $d u$ sens concret du verbe. Pourtant, d'après l'observation du corpus d'experts, le sens par extension/abstrait est le plus employé dans l'écrit scientifique. Ceci rejoint notre constat sur l'importance de la maitrise du LST et de l'emploi des patrons verbaux de ce lexique. Nous remarquons également que certaines lacunes (notamment lexicales) propres aux étudiants non-natifs leur rendent la maîtrise des patrons encore plus complexe.

113 Sur le plan didactique, nous proposons des pistes de réflexions pour enseigner les patrons verbaux, à travers l'approche inductive et en se basant sur les corpus, afin d'améliorer l'enseignement/apprentissage de ces constructions essentielles dans l'écrit scientifique.

\section{BIBLIOGRAPHIE}

Biber, D. (1993). Using register-diversified corpora for general language studies. Computational linguistics, 19(2), 219-241.

Blumenthal, P. (2008). Combinatoire des prépositions: approche quantitative. Langue française, 157 (1), 37. http://doi.org/10.3917/lf.157.0037 [25/02/2016]

Boch, F., Buson, L. 2012. « Orthographe \& grammaire à l'université. Quels besoins ? Quelles démarches pédagogiques? ». Scripta, vol. 16, n³0, p. 31-51.

Cavalla, C. (2014). Collocations transdisciplinaires d'apprentissage : réflexions pour l'enseignement. In : González-Rey Isabel (éd.) : Outils et méthodes d'apprentissage en phraséodidactique : 151-169. Éditions Modulaires Européennes.

Chartrand, S.-G. 1996. « Apprendre la grammaire par la démarche active de découverte ». In : Pour un nouvel enseignement de la grammaire, $\mathrm{n}^{\circ} 2$, p. 19-225.

Colletta, J.-M. 2004. Le développement de la parole chez l'enfant âgé de 6 et 11 ans : corps, langage et cognition. Sprimont: Editions Mardaga.

Drouin, P. (2007). Identification automatique du lexique scientifique transdisciplinaire. Revue française de linguistique appliquée, vol. XII(2), 4564.

Dubois, J., \& Dubois-Charlier, F. (1997). Les verbes français. Larousse. 
Dubois, J., \& Dubois-Charlier, F. (2010). La combinatoire lexico-syntaxique dans le Dictionnaire électronique des mots. Les termes du domaine de la musique à titre d'illustration. Langages, (3), 31-56.

Flaux, N., \& Van de Velde, D. (2000). Les noms en français : esquisse de classement. Éditions Ophrys. Hanks, P. (2013). Lexical Analysis : Norms and Exploitations. Mit Press.

Kupsc, A. (2007). Extraction automatique de cadres de sous-catégorisation verbale pour le français à partir d'un corpus arboré. TALN 2007.

Messiant, C., Gábor, K., \& Poibeau, T. (2010). Acquisition de connaissances lexicales à partir de corpus : la sous-catégorisation verbale en français. Traitement automatique des langues, 51(1), 65-96. Nazar, R., \& Renau, I. (à paraître). A quantitative analysis of the semantics of verb-argument structures.

Paquot, M. (2010). Academic vocabulary in learner writing : From extraction to analysis. Bloomsbury Publishing.

Paquot, M., \& Bestgen, Y. (2009). Distinctive words in academic writing : A comparison of three statistical tests for keyword extraction. Language and Computers, 68(1), 247269.

Renau, I., \& Paz, B. (2011). The Spanish Learner's Dictionary DAELE on the Panorama of the Spanish E-lexicography. In : I., Kosem \& K., Kosem (eds.) : Electronic lexicography in the 21st century : new applications for new users : proceedings of eLex 2011, 10-12 November 2011, Bled, Slovenia, Ljubljana : Trojina, Institute for Applied Slovene Studies, 221-226.

Rinck, F. 2010. Réflexivité et écrits de recherche. Propositions pour une formation universitaire à et par la littéracie. Dans De la maîtrise du français aux littéracies dans l'enseignement supérieur (Actes des journées d'études du Centre de Méthodologie Universitaire " CMU 2010 ") (p. 79-91). Belgique.

Rinck, F. (2013). L'article de recherche en sciences humaines: approche en corpus. In Séminaire des Aspects concrets de la thèse, EHESS. Paris, France. Consulté à l'adresse http://halshs.archivesouvertes.fr/halshs-00998105 [25/02/2016]

Sinclair, J. (1991). Corpus, concordance, collocation (Vol. 1). Oxford University Press Oxford. Tognini-Bonelli, E. (2001). Corpus linguistics at work (Vol. 6). John Benjamins Publishing.

Tutin, A. (2007). Autour du lexique et de la phraséologie des écrits scientifiques. Revue française de linguistique appliquée, vol. XII(2), 55.

Tutin, A., \& Grossmann, F. (Éd.). (2014). L'Écrit scientifique : du lexique au discours. Autour de Scientext . Presses universitaires de Rennes.

Williams, G., \& Million, C. \& Alonso, A. (2012). Growing naturally : The DicSci Organic E-Advanced Learner's Dictionary of Verbs in Science. Proceedings of the 15th EURALEX International Congress. University of Oslo.

\section{NOTES}

1. « verbs that serve specific rhetorical or organizational functions in academic prose generally enter compositional and flexible sequences. "

2. Hatier, S., Augustyn, M., Tran, T.T.H., Yan, R., Tutin, A., \& Jacques, M-P. (2015). French crossdisciplinary scientific lexicon : extraction and linguistic analysis - article soumis 
3. Nous avons pour une étude précédente constitué un corpus de grande taille du français (120 millions de mots) nous servant de corpus de contraste pour le calcul de spécificité

4. Dubey Gérard, "Nouvelles techniques d'identification, nouveaux pouvoirs. Le cas de la biométrie », Cahiers internationaux de sociologie 2/2008 ( $\left.n^{\circ} 125\right)$, p. 263-279

5. Les profils combinatoires sont, selon Blumenthal (2008), "l'image que donne du comportement d'un mot de base l'ensemble de ses collocatifs ".

6. Correspondant à l'acception 6 selon le Dictionnaire Électronique des Mots (Dubois \& DuboisCharlier, 2010), consulté le 11/01/2016 à l'adresse: http://rali.iro.umontreal.ca/LVF+1/ alphabetique/P.html\#presenter

7. Wolff, M. (2003). 7. Apports de l'analyse géométrique des données pour la modélisation de l'activité. Le Travail humain, 1, 195-227.

8. Mémoires de 60 pages environ, hors annexes.

9. Corpus constitué par Rinck, F. Boch, F, \& Jacques, M-P, disponible à l'adresse suivante : http:// lidilem.u-grenoble3.fr/ressources/corpus-du-labo/article/corpus-litteracie-avancee (consulté le 11/01/2016).

10. Dont un descriptif est consultable à l'adresse suivante : http://scientext.msh-alpes.fr/

11. Anthropologie, économie, géographie, histoire, linguistique, psychologie, sciences de l'éducation, sciences politiques, sciences de l'information, sociologie

12. Ait-Mokthar, S., \& Chanod, J. P. (2002). Robustness beyond Shallowness: Incremental Dependency Parsing. Special issue of NLE Journal.

13. [u3_advmod], [u3_de_vmod] et [subj] réfèrent aux étiquettes de relations de dépendances issues de l'analyseur et de nos grammaires locales.

14. À l'aide du calcul de rapport de vraisemblance.

15. Un patron est une déclaration de la structure de proposition (valence) associée à un sens d'un verbe, avec les valeurs sémantiques typiques de chaque argument qui se réalisent par des cooccurrences saillantes [...] Les différentes valeurs sémantiques des arguments activent les différents sens de chaque verbe.

16. http://www.pdev.org.uk/\#browse?q=;f=c. Ce dictionnaire est encore en cours de construction, 1348 verbes en anglais y sont répertoriés et l'objectif est d'atteindre 5396 verbes.

17. http://www.iula.upf.edu/rec/daele/

18. http://olivier.kraif.u-grenoble3.fr/anaText/index.php

19. Nous conservons dans les exemples les graphies d'origine présentes dans les corpus

\section{RÉSUMÉS}

Nous présentons dans cet article une étude des patrons verbaux employés par des scripteurs étudiants (apprenants du français et natifs) et par des scripteurs experts (chercheurs). Nous nous intéressons ici à l'emploi de verbes fréquents dans l'écrit scientifique (montrer, considérer, décrire, expliquer) par les scripteurs étudiants. Nous analysons, pour ces derniers, les constructions verbales utilisées préférentiellement ainsi que celles qui leur posent le plus de problèmes. Pour ce faire, en nous basant sur des corpus analysés en dépendances, nous extrayons semiautomatiquement les patrons verbaux les plus fréquents et analysons les points de convergence et de divergence entre les différents types de scripteurs. 
This study focuses on the comparison between verbal patterns used in academic writing by students (native and French learners) and expert writers (researchers). We aim at identifying how students use verbs that frequently appear in academic writing (montrer / show, considérer / consider, décrire / describe, expliquer / explain). Our objective is to pinpoint verbal constructions preferentially used by students and verbal constructions they do not master. Using syntactically parsed corpora of scientific papers and dissertations, we collect and modelize lexico-syntactic patterns for investigated verbs. Thus we analyze shared and specific verbal patterns between student and expert writers.

\section{INDEX}

Mots-clés : écrit scientifique, sous-catégorisation verbale, linguistique de corpus, patron lexicosyntaxique

Keywords : academic writing, verbal subcategorization, corpus linguistics, lexico-syntactic pattern

\section{AUTEURS}

\section{SYLVAIN HATIER}

LIDILEM, Univ. Grenoble Alpes Sylvain.Hatier@u-grenoble3.fr

\section{RUI YAN}

LIDILEM, Univ. Grenoble Alpes

Rui.Yan@u-grenoble3.fr 